\title{
Passive synchronized Q-switching between a quasi-three-level and a four-level laser
}

Cheng, Haynes Pak Hay; Tidemand-Lichtenberg, Peter; Jensen, Ole Bjarlin; Andersen, Peter E.; Petersen, Paul Michael; Pedersen, Christian

\section{Published in:}

Proceedings of SPIE - The International Society for Optical Engineering

Link to article, DOI:

$10.1117 / 12.873382$

Publication date:

2011

Document Version

Publisher's PDF, also known as Version of record

Link back to DTU Orbit

Citation (APA):

Cheng, H. P. H., Tidemand-Lichtenberg, P., Jensen, O. B., Andersen, P. E., Petersen, P. M., \& Pedersen, C. (2011). Passive synchronized Q-switching between a quasi-three-level and a four-level laser. Proceedings of SPIE - The International Society for Optical Engineering, 7912, 79120W. https://doi.org/10.1117/12.873382

\section{General rights}

Copyright and moral rights for the publications made accessible in the public portal are retained by the authors and/or other copyright owners and it is a condition of accessing publications that users recognise and abide by the legal requirements associated with these rights.

- Users may download and print one copy of any publication from the public portal for the purpose of private study or research.

- You may not further distribute the material or use it for any profit-making activity or commercial gain

- You may freely distribute the URL identifying the publication in the public portal 


\title{
Passive synchronized Q-switching between a quasi-three-level and a four-level laser
}

\author{
Haynes P. H. Cheng*, Peter Tidemand-Lichtenberg, Ole B. Jensen, Peter E. Andersen, \\ Paul Michael Petersen, Christian Pedersen \\ DTU Fotonik, Technical University of Denmark, Frederiksborgvej 399, DK-4000, Roskilde, \\ Denmark
}

\begin{abstract}
Synchronized Q-switching between quasi-three-level and four-level lasers is interesting for sum-frequency generation into the blue and ultraviolet. We report, for the first time, stable synchronized Q-switching between a quasi-three-level laser at $946 \mathrm{~nm}$ and a four-level laser at $1064 \mathrm{~nm}$ in an all passive approach. While timing jitter of the individual freerunning lasers were on the order of $10 \mu \mathrm{s}$, the relative timing jitter, defined as one standard-deviation of the experimental data, was only 9 ns between the two synchronized pulses. The minimum delay between the two pulses was 64 ns during stable operation, which gave a $79 \%$ temporal overlap when normalized against the zero-delay scenario. Preliminary results show promise for non-linear frequency conversion, which could lead to high power pulsed blue and ultraviolet lasers.
\end{abstract}

Keywords: Lasers, Q-switched, diode-pumped, solid-state, Nd:YAG

\section{INTRODUCTION}

Synchronized pulsing of different wavelength lasers is by no means a new concept. In fact, simultaneous dualwavelength operation of a ruby laser was reported by Schawlow and Devlin ${ }^{1}$ just seven months after Maiman's demonstration of the world's first laser. In 1965, Caviello et al. demonstrated synchronized Q-switching between the $694.3 \mathrm{~nm}$ and $692.9 \mathrm{~nm}$ line in a ruby laser².

The main motivation for synchronized Q-switching in early works were in long distance interferometery ${ }^{3}$ and in power scaling $^{4}$; in recent years, however, considerable interests have been placed on non-linear frequency mixing, which implies that the lasers should be able to operate at fairly different wavelengths in order to cover a wide spectral range. Fig. 1 shows the possible wavelengths that is covered by sum frequency mixing the various transitions of Nd:YAG, including the quasi-three-level transitions at $939 \mathrm{~nm}$ and $946 \mathrm{~nm}$.

(a)

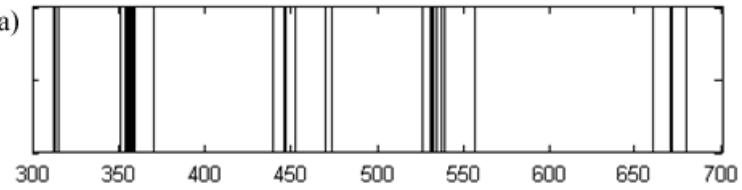

(b)

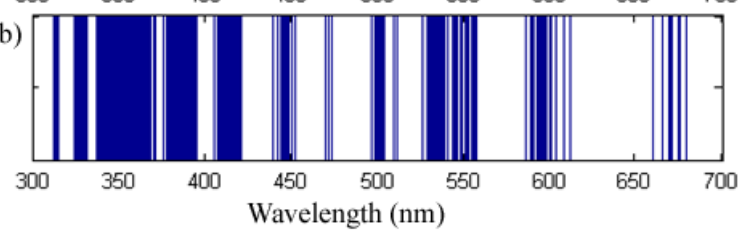

(c)

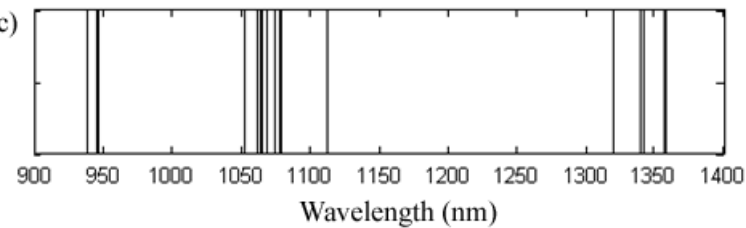

Figure 1. Spectral range of non-linear frequency mixing covered by Nd:YAG up to the third harmonic. (a) only the degenerate wavelengths of $2 \omega$ and $3 \omega$, (b) possibilities using sum-frequency mixing, (c) fundamental wavelengths including the quasi-three-level transitions at $939 \mathrm{~nm}$ and $946 \mathrm{~nm}$.

*hach@fotonik.dtu.dk; phone +45 4677 5418; www.fotonik.dtu.dk

Solid State Lasers XX: Technology and Devices, edited by W. Andrew Clarkson, Norman Hodgson, Ramesh Shori, Proc. of SPIE Vol. 7912, 79120W · (c) 2011 SPIE · CCC code: 0277-786X/11/\$18 · doi: 10.1117/12.873382 
The main difficulty in synchronization of Q-switched lasers with significantly different wavelengths lies in compensating for their respective different gain cross-sections. In Nd:YAG, for example, the gain cross-section of the $946 \mathrm{~nm}$ transition is ten times less than that at $1064 \mathrm{~nm}^{5,6}$. In addition, if one of the lasers is a quasi-three-level system, the effect of gain difference is further worsened due to reabsorption.

Using an active approach, Herault et al. synchronized a quasi-three-level laser at $912 \mathrm{~nm}$ and a four-level laser at 1063 $\mathrm{nm}$ by adjusting the opening times of the two active Q-switches ${ }^{7}$. By making the $912 \mathrm{~nm}$ pulse relatively long, a good temporal overlap was achieved. The authors reported a timing jitter of 40 ns and subsequently generated $9 \mathrm{~kW}$ of peak power at $491 \mathrm{~nm}$ through non-linear cavity dumping.

To the authors' knowledge, there has only been one publication on passively synchronized Q-switching between a quasithree-level laser and a four-level laser. Zhang et al. obtained simultaneous dual-wavelength operation at $1064 \mathrm{~nm}$ and $946 \mathrm{~nm}$ by introducing a relatively large loss on the $1064 \mathrm{~nm}$ laser. Unfortunately, the authors did not report on the temporal characteristics of the synchronized pulses and reported fluctuations in the output power ${ }^{8}$.

In this paper, two separately pumped Nd:YAG laser crystals were used to equalize the repetition rates at $946 \mathrm{~nm}$ and $1064 \mathrm{~nm}$ respectively, and stable synchronization is reported for the first time. A single Cr:YAG saturable absorber (SA), placed in a common section of the two cavities, was used to synchronize the two pulse trains. Synchronization is obtained by careful mode matching of the two lasers in the SA, and controlling the pump power of each laser individually. The relative timing jitter between the two pulses was $9 \mathrm{~ns}$, which is defined as the root-mean-square deviation from the mean delay, or one standard deviation of the experimental data. A minimum delay of 64 ns was observed between the two pulse trains, and by making one pulse relatively long when compared to the other, a $79 \%$ temporal overlap was achieved compared to the zero-delay scenario.

\section{EXPERIMENT}

\subsection{Experimental Setup}

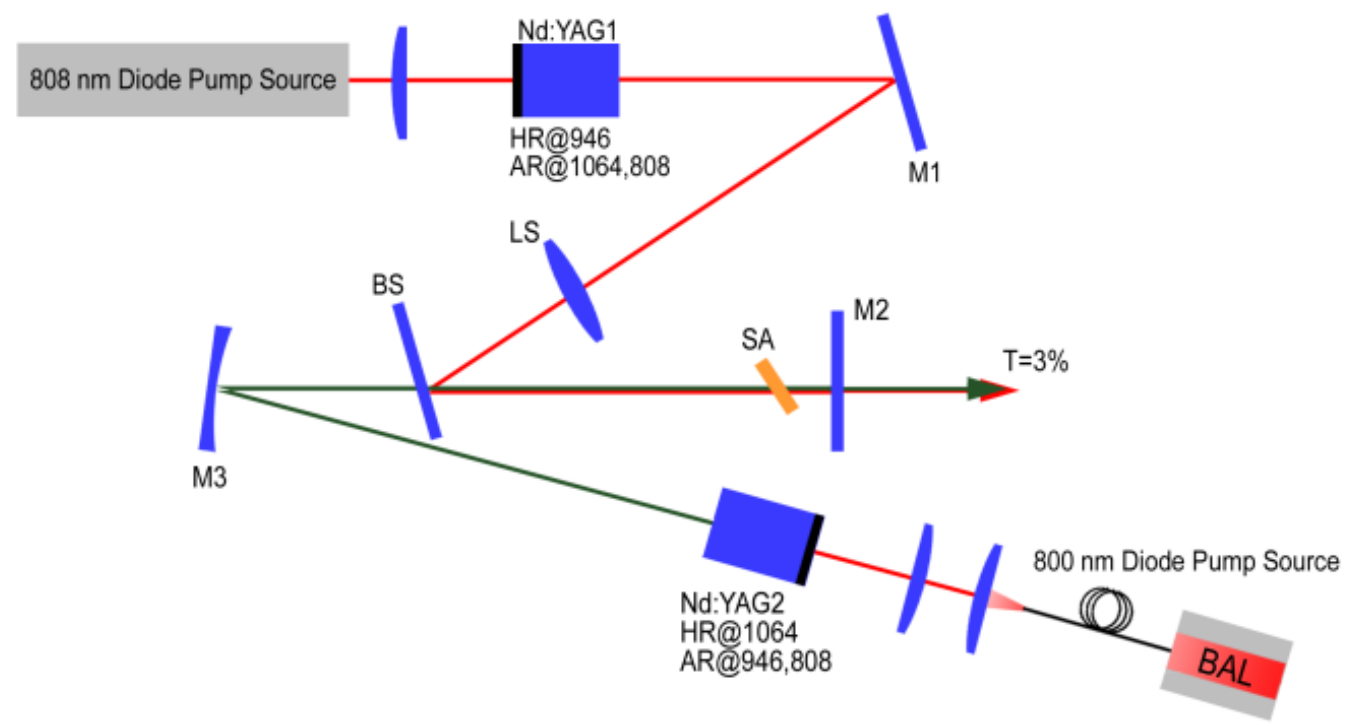

Figure 2. Experimental setup for a Y-shaped cavity with a shared Q-switch between the $946 \mathrm{~nm}$ and $1064 \mathrm{~nm}$ lasers. Nd:YAG1 and Nd:YAG2 are the alser crystals for $946 \mathrm{~nm}$ and $1064 \mathrm{~nm}$, respectively. SA is the saturable absorber.

Fig. 2 illustrates the experimental setup. The HR facet of Nd:YAG1, mirrors M1, BS and M2 form the $946 \mathrm{~nm}$ cavity, while the HR facet of Nd:YAG2, mirrors M3 and M2 form the $1064 \mathrm{~nm}$ cavity. The SA is a $0.97 \mathrm{~mm}$ Brewster-cut Cr:YAG specified for $85 \%$ unsaturated transmission at $1064 \mathrm{~nm}$ at normal incidence. Taking into account the SA is oriented at Brewster's angle and using the absorption cross-sections published by Zhang et al. ${ }^{9}$, the unsaturated transmission at $1064 \mathrm{~nm}$ and $946 \mathrm{~nm}$ are calculated to be $82 \%$ and $83 \%$, respectively. BS is a dichroic mirror used to couple the $1064 \mathrm{~nm}$ beam into the $946 \mathrm{~nm}$ laser cavity, while mirror M2 acts as the output coupler for both lasers. M2 is coated for 3\% transmission at both $1064 \mathrm{~nm}$ and $946 \mathrm{~nm}$. 
The mode sizes of the two lasers are shown in table 1 below:

Table 1. Experimental mode sizes for the $946 \mathrm{~nm}$ and $1064 \mathrm{~nm}$ lasers.

\begin{tabular}{|l|c|c|}
\hline & $\mathbf{9 4 6} \mathbf{~ n m}$ & $\mathbf{1 0 6 4} \mathbf{~ n m}$ \\
\hline $\begin{array}{l}\text { Pump diode mode size inside } \\
\text { laser crystal }\end{array}$ & $59 \mu \mathrm{m}(\mathrm{h}) \times 80 \mu \mathrm{m}(\mathrm{v})$ & $70 \mu \mathrm{m}$ (in both directions) \\
\hline $\begin{array}{l}\text { Cavity mode size inside laser } \\
\text { crystal }\end{array}$ & $77 \mu \mathrm{m}$ (in both directions) & $88 \mu \mathrm{m}(\mathrm{h}) \times 108 \mu \mathrm{m}(\mathrm{v})$ \\
\hline Cavity mode size inside SA & $24 \mu \mathrm{m}$ (in both directions) & $72 \mu \mathrm{m}(\mathrm{h}) \times 97 \mu \mathrm{m}(\mathrm{v})$ \\
\hline
\end{tabular}

For efficient Q-switching in a quasi-three-level laser, two operating conditions should be met: 1) good overlap between the pump and cavity modes inside the laser crystal. 2) a small cavity mode inside the passive Q-switch to achieve efficient bleaching. These imply that the $946 \mathrm{~nm}$ laser cavity is most efficient near the stability limit, where stability of the cavity itself is very sensitive to perturbation. Thus, an intracavity lens was used instead of a curved folding mirror to avoid astigmatism, which would have further reduced the stability region. The intracavity lens, LS, has a focal length of $75 \mathrm{~mm}$ and an insertion loss of $1.9 \%$.

Following the method used by Herault et al. ${ }^{7}$, the $946 \mathrm{~nm}$ laser cavity in Fig. 2 was made relatively long to ensure a good temporal overlap between the two pulses. The $946 \mathrm{~nm}$ cavity length was $37 \mathrm{~cm}$, while the 1064 nm cavity length was 19 $\mathrm{cm}$. These resulted in $200 \mathrm{~ns}$ and $45 \mathrm{~ns}$ FWHM pulse widths for the $946 \mathrm{~nm}$ and $1064 \mathrm{~nm}$ lasers, respectively.

Throughout the experiments presented here, the incident pump power of the $946 \mathrm{~nm}$ laser was held fixed at $1.6 \mathrm{~W}$, while the pump power of the $1064 \mathrm{~nm}$ laser was varied. Detailed pump source specifications can be found in a separate publication $^{10}$.

\subsection{Results and Discussion}

Stable locking of the two pulses was observed over a wide range of pump powers. Fig. 3 illustrates the repetition rates of the two lasers. As a base-line comparison, repetition rates of the two individual free-running lasers were first measured with the other laser switched off. These are plotted in dashed lines.

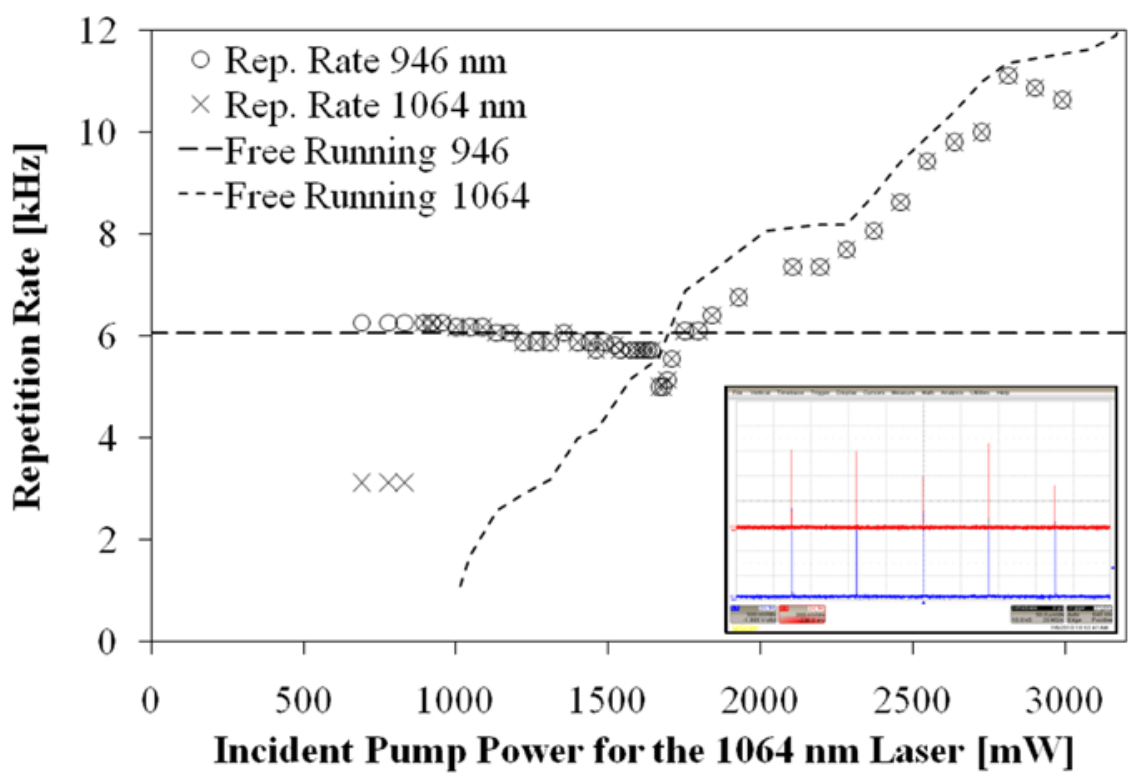

Figure 3. Repetition rates of the synchronized system and of the respective free-running lasers at $946 \mathrm{~nm}$ and 1064 $\mathrm{nm}$. Incident pump power of the $946 \mathrm{~nm}$ laser was held fixed at $1.6 \mathrm{~W}$ while the pump power of the $1064 \mathrm{~nm}$ laser was varied. Inset shows oscilloscope traces of the two lasers at $11 \mathrm{kHz}$. 
The repetition rates of the synchronized lasers are plotted in circles and crosses for the $946 \mathrm{~nm}$ laser and $1064 \mathrm{~nm}$ laser, respectively. It can be clearly seen that the repetition rates of the two lasers are stably locked up to $11 \mathrm{kHz}$, almost twice the free-running repetition rate of the $946 \mathrm{~nm}$ laser. The corresponding oscilloscope trace at $11 \mathrm{kHz}$ is shown in the inset on the lower right-hand corner. The first three data points in Fig. 3 indicate 2:1 synchronization, where a $1064 \mathrm{~nm}$ laser pulse is generated with every other $946 \mathrm{~nm}$ pulse. This is due to the relatively low gain at low pump powers and is in agreement with previous publications ${ }^{11,12}$.

It should be noted that when the free-running repetition rates of the two lasers are equal, the synchronization becomes unstable. The unstable regime is narrow, within tens of $\mathrm{mW}$ of incident pump power, and will be described later in the manuscript.

It can be seen in Fig. 3 that the synchronized lasers operate at the higher repetition rate of the two lasers: when the 1064 $\mathrm{nm}$ free-running repetition rate is below that of the $946 \mathrm{~nm}$ laser, the synchronized lasers operate at the free-running repetition rate of the $946 \mathrm{~nm}$ laser; when the $1064 \mathrm{~nm}$ free-running repetition rate is above that of the $946 \mathrm{~nm}$ laser, the synchronized lasers operate at the free-running repetition rate of the $1064 \mathrm{~nm}$ laser. This can be explained as follows: the higher-repetition rate laser (master) reaches threshold sooner than the lower-repetition rate laser (slave), and once the master laser bleaches the SA, the slave laser reaches threshold as well and is able to emit a pulse synchronously. Thus, the slave laser is able to emit its pulses at an earlier time than it would have otherwise, reaching the same repetition rate as the master laser.

Since the slave laser only starts to build up after the SA is bleached by the master laser, a small delay between the two pulses is observed when the free-running repetition rates of the two lasers are not equal. This is plotted in Fig. 4 below. A positive delay indicates the $1064 \mathrm{~nm}$ laser is leading the $946 \mathrm{~nm}$ laser, while a negative delay indicates it is lagging the $946 \mathrm{~nm}$ laser. The switching point at $1.6 \mathrm{~W}$, when the $1064 \mathrm{~nm}$ laser becomes the master laser, corresponds to the point when the free-running repetition rates of the two lasers are equal.

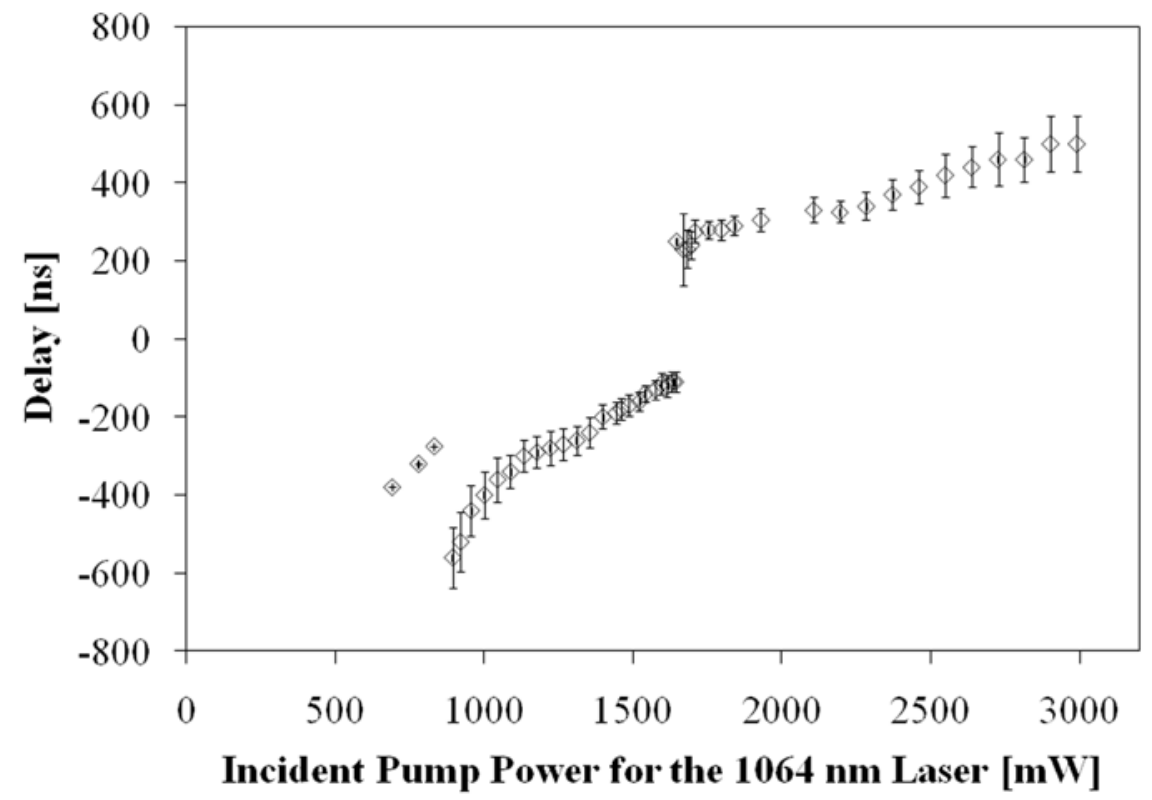

Figure 4. Relative delay between the two pulses as a function of the $1064 \mathrm{~nm}$ laser pump power. Error bars show the 6-standard deviation jitter measurement over 1000 pulses. No jitter measurements were made for the first three data points, where a $1064 \mathrm{~nm}$ pulse arrives only with every other $946 \mathrm{~nm}$ pulse.

As mentioned above, the synchronization is unstable at the switching point. To better understand the dynamics of the synchronization, particularly in this unstable regime, the coupled rate equations ${ }^{12}$ was adapted to include a three-level system, and was used to qualitatively model the temporal dynamics of the synchronized lasers. The one-dimensional model assumes that the photons are evenly distributed throughout the laser cavity, and focuses on the temporal characteristic of the synchronized system. The pulse build-up (output power) and cavity losses are shown qualitatively as 
functions of time in Fig. 5 (a) and (b). The $1064 \mathrm{~nm}$ laser absorbed pump power is increased by $100 \mathrm{~mW}$ in Fig. 5 (b), while all other parameters were fixed. Dashed lines indicate the lasers are below threshold.

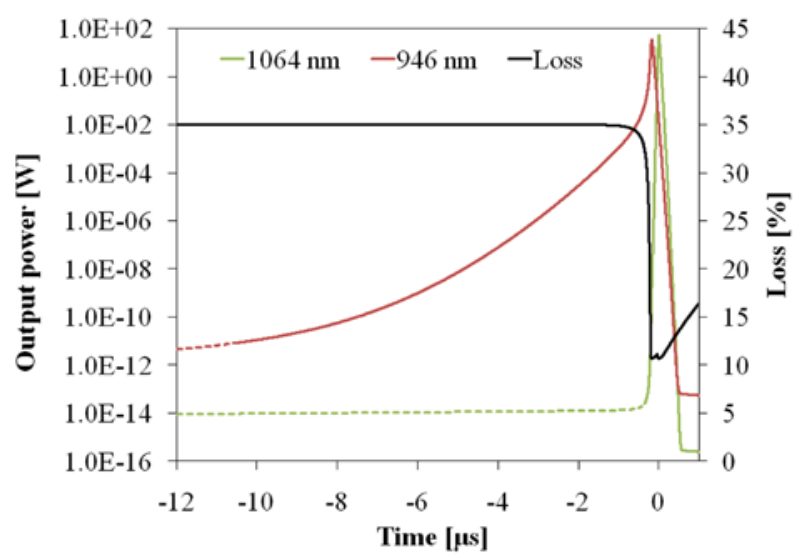

(a)

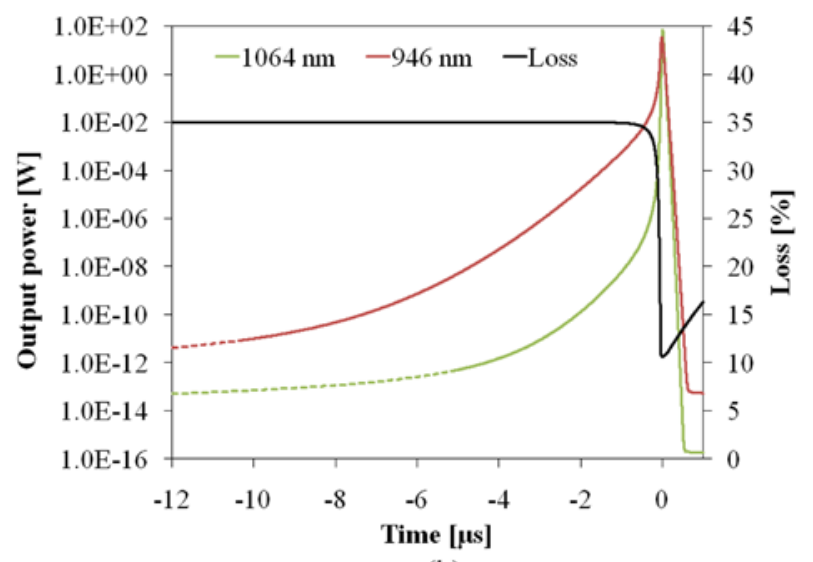

(b)

Figure 5. Qualitative plots of the pulse build-up (output power) and cavity losses for (a) when the 946 nm laser is the master laser and (b) when the free-running repetition rates of the two lasers are equal.

It can be seen that when the $946 \mathrm{~nm}$ laser is the master laser (Fig. 5 (a)), the point at which the $1064 \mathrm{~nm}$ laser reaches threshold is well defined; particularly, when the SA is bleached. The instantaneous excess gain in the $1064 \mathrm{~nm}$ laser is then directly related to the pumping rate. This explains the dependence of the delay on the $1064 \mathrm{~nm}$ laser pump power as the pump power and excess gain is increased, the $1064 \mathrm{~nm}$ laser build-up time, and hence, also the delay, is decreased. A similar argument could be made for when the $1064 \mathrm{~nm}$ laser is the master laser.

If the repetition rates of the two lasers are matched (Fig. 5 (b)), both lasers reach threshold before the SA is bleached. As a result, both lasers are building up rapidly due to stimulated emission. In this case, small gain fluctuations that advance or delay the threshold of either laser, on the order of microseconds, can have a critical impact on the timing of the two pulses. Thus, the $946 \mathrm{~nm}$ pulse would build-up first at times, while the $1064 \mathrm{~nm}$ pulse would build-up first at others. This leads to a significantly large timing jitter at the switching point. The error bars in Fig. 4 show the relative timing jitter between the two pulses observed experimentally. Following the convention by laser manufacturers and previous publications $^{13,14}$, the jitter is defined as the root-mean-square deviation from the mean delay at a particular pump level. In Fig. 4, however, the error bars are drawn to indicate \pm 3 standard deviations so that they can be visible. The minimum jitter observed was $9 \mathrm{~ns}$, and the minimum delay for stable operation depends effectively on the timing jitter of the individual free-running lasers ${ }^{15}$. As mentioned above, the unstable operating regime was observed to be very narrow.

To investigate the minimum delay that can be obtained with the current setup, the $1064 \mathrm{~nm}$ cavity mode size inside the SA is reduced to match that of the $946 \mathrm{~nm}$ laser in the horizontal direction (to $24 \mu \mathrm{m}(\mathrm{h}) \mathrm{x} 80 \mu \mathrm{m}(\mathrm{v})$ ). The minimum delay between the two pulses was reduced to $64 \mathrm{~ns}$ when the $946 \mathrm{~nm}$ laser was the master laser. Fig. 6 shows the corresponding oscilloscope traces, which contains 200 traces superimposed with the same trigger reference point. The jitter in this case was $12 \mathrm{~ns}$. The temporal overlap, defined as the product of the two signals integrated over the pulse duration, was found to be $79 \%$ when normalized against the zero-delay scenario. The focusing lens of the $1064 \mathrm{~nm}$ laser pump beam was changed from $40 \mathrm{~mm}$ to $20 \mathrm{~mm}$ to suppress higher order modes. Further reduction of the $1064 \mathrm{~nm} \mathrm{SA}$ mode size was not possible due to physical limits with the current setup. 


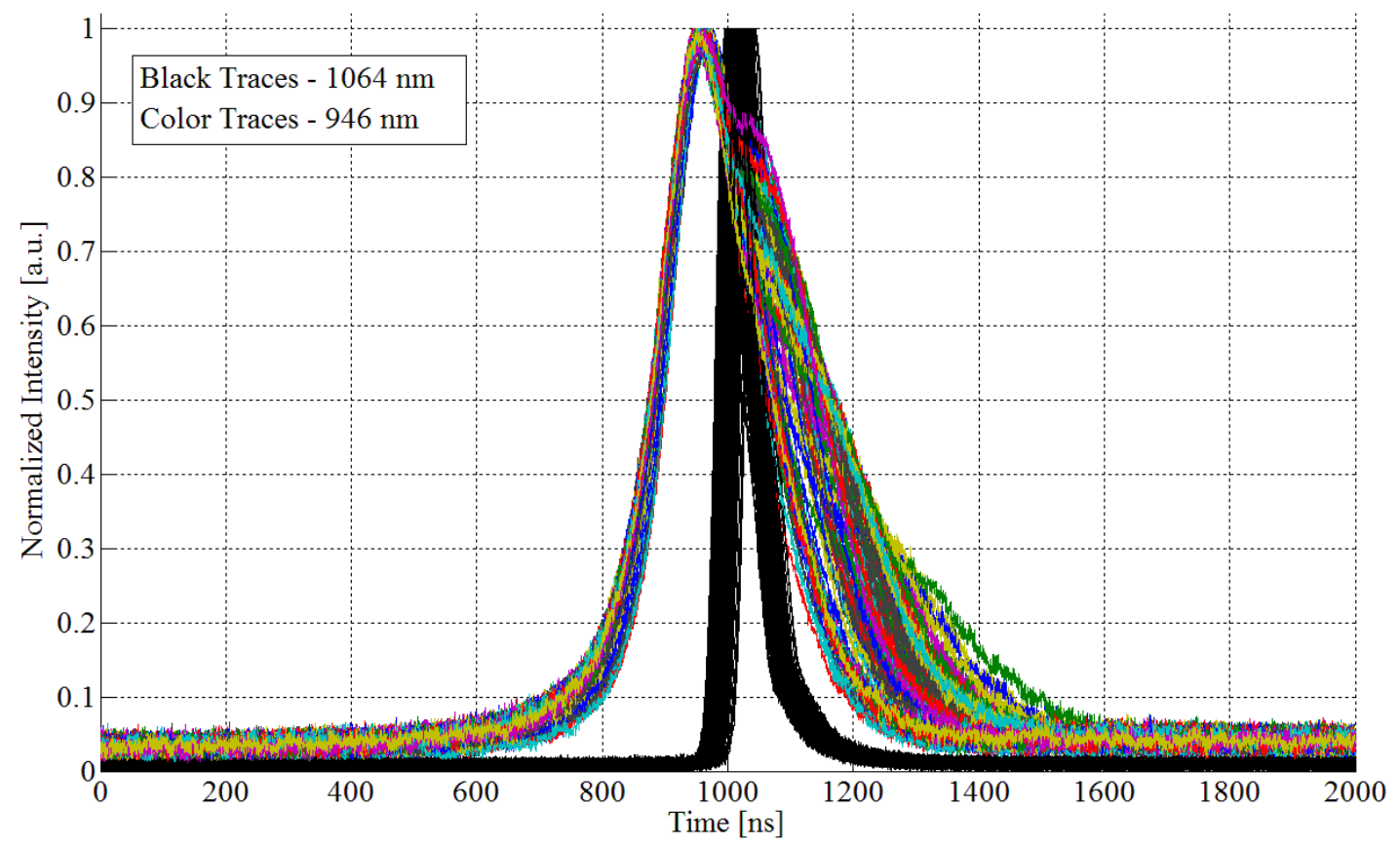

Figure 6. Superposition of 200 oscilloscope traces of the two laser pulses. Color traces show the $946 \mathrm{~nm}$ pulses while the black traces show the $1064 \mathrm{~nm}$ pulses.

\section{CONCLUSION}

We demonstrated for the first time, stable synchronized Q-switching between a quasi-three-level laser and a four-level laser using a passive Q-switch. A numerical model shows the temporal dynamics of the synchronized system, the origin of the delay between the two pulses, and the instability when the repetition rates of the two lasers are equal. Timing jitter at the best operating point was observed to be 9 ns, which is comparable to a previously published active system ${ }^{7}$. By better matching the mode sizes inside the SA and making one pulse relatively long when compared to the other, a temporal overlap of $79 \%$ was obtained when normalized against the zero-delay scenario. The stability and temporal overlap reported here show potential for applications in sum-frequency generation.

\section{ACKNOWLEDGEMENT}

The authors gratefully acknowledge Ferdinand-Braun-Institut, Leibniz Institut für Höchstfrequenztechnik for providing the tapered diode laser pump source, and the financial support of the European Community through the FP-6 project WWW.BRIGHTER.EU contract IST-2005-035266.

\section{REFERENCES}

[1] Shawlow, A. L. and Devlin, G. E., "Simultaneous optical maser action in two ruby satellite lines," Phys. Rev. Lett. 6(3), 96-98 (1961).

[2] Calviello, J. A., Fisher, E. W. and Heller, Z. H., "Simultaneous Laser Oscillation at R1 and R2 Wavelengths in Ruby,” IEEE J. Quantum Electron. QE1(3), 132 (1965).

[3] Bennett, W. R. and Knutson, J. W., "Simultaneous Laser Oscillation on the Neon Doublet at $1.1523 \mu$," in Proc. IEEE 52(7), 861-862 (1964).

[4] Gregg, D. W. and Thomas, S. T., "Simultaneous Giant Pulses from Five Ruby Laser Oscillators,” J. Appl. Phys. 37(10), 3750-3753 (1966). 
[5] Aull, B. F. and Jenssen, H. P., "Vibronic interactions in Nd:YAG resulting in nonreciprocity of absorption and stimulated emission cross sections,” IEEE J. Quantum Electron. QE18(5), 925-930 (1982).

[6] Koechner, W., [Solid-State Laser Engineering 6th ed.], Springer, Round Hill, VA, 55-61 (2006).

[7] Herault, E., Lelek, M., Balembois, F. and Georges, P., "Pulsed blue laser at $491 \mathrm{~nm}$ by nonlinear cavity dumping," Opt. Express 16(24), 19419-19426 (2008).

[8] Zhang, L., Wei, Z., Feng, B., Li, D. and Zhang, Z., "Simultaneous dual-wavelength Q-switched Nd:YAG laser operating at $1.06 \mu \mathrm{m}$ and $946 \mathrm{~nm}$,” Opt. Commun. 264(1), 51-54 (2006).

[9] Zhang, X., Brenier, A., Wang, J. and Zhang, H., “Absorption cross-sections of Cr4+:YAG at 946 and 914 nm,” Opt. Mater. 26(3), 293-296 (2004).

[10] Cheng, H. P. H., Tidemand-Lichtenberg, P., Jensen, O.B., Andersen, P. E., Petersen, P. M. and Pedersen, C., "All passive synchronized Q-switching of a quasi-three-level and a four-level Nd:YAG laser,” Opt. Express 18(23), 23987-23993 (2010).

[11] Tidemand-Lichtenberg, P., Janousek, J., Melich, R., Mortensen, J. L. and Buchhave, P., "Synchronization of 1064 and $1342 \mathrm{~nm}$ pulses using passive saturable absorbers,” Opt. Commun. 241(4-6), 487-492 (2004).

[12] Janousek, J., Tidemand-Lichtenberg, P., Mortensen, J. L. and Buchhave, P., "Investigation of passively synchronized dual-wavelength Q-switched lasers based on V:YAG saturable absorber,” Opt. Commun. 265(1), 277282 (2006).

[13] Cole, B., Goldberg, L., Trussel, C. W., Hays, A., Schilling, B. W. and McIntosh, C., "Reduction of timing jitter in a Q-switched Nd:YAG laser by direct bleaching of a $\mathrm{Cr}^{4+}$ :YAG saturable absorber,” Opt. Express 17(3), 1766-1771 (2009).

[14] Steinmetz, A., Nodop, D., Martin, A., Limpert, J. and Tünnermann, A., "Reduction of timing jitter in passively Qswitched microchip lasers using self-injection seeding,” Opt. Lett. 35(17), 2885-2887 (2010).

[15] Cheng, H. P. H., Tidemand-Lichtenberg, P., Jensen, O.B., Andersen, P. E., Petersen, P. M. and Pedersen, C., "Experimental investigation of relative timing jitter in passively synchronized Q-switched lasers," Opt. Lett. (under review). 